\title{
Osteochondral Live Slices as Physiological in-vitro Model of the Human Joint for High-throughput Applications
}

Jacob Spinnen ( $\nabla$ jacob.spinnen@charite.de)

Laboratory for Tissue Engineering BIH Center for Regenerative Therapies \& Department for Rheumatology and Clinical Immunology Charité - Universitätsmedizin Berlin

\section{Carsten Rendenbach}

Charite Universitatsmedizin Berlin Campus Charite Mitte: Charite Universitatsmedizin Berlin

\section{Anja Kühl}

Charite Universitatsmedizin Berlin Campus Charite Mitte: Charite Universitatsmedizin Berlin

\section{Ufuk Sentürk}

Charite Universitatsmedizin Berlin Campus Charite Mitte: Charite Universitatsmedizin Berlin

\section{Daniel Kendoff}

HELIOS Hospital Berlin-Buch: HELIOS Klinikum Berlin-Buch

\section{Henrik Mei}

Deutsches Rheuma-Forschungszentrum Berlin

\section{Michael Sittinger}

Laboratory for Tissue Engineering BIH Center for Regenerative Therapies \& Department for Rheumatology and Clinical Immunology Charité - Universitätsmedizin Berlin Charitéplatz 1, 10117 Berlin Tilo Dehne

Charite Universitatsmedizin Berlin Campus Charite Mitte: Charite Universitatsmedizin Berlin

\section{Research article}

Keywords: Osteoarthritis, osteochondral live-slices, explant culture, joint modelling

Posted Date: September 4th, 2020

DOI: https://doi.org/10.21203/rs.3.rs-68789/v1

License: (c) (1) This work is licensed under a Creative Commons Attribution 4.0 International License. Read Full License 


\section{Abstract}

Background: Current advances in musculoskeletal research yielded numerous new insights and therapy options for diseases of the joints but preclinical in-vitro testing is currently limited to self-manufactured 2D and 3D cell cultures only remotely mimicking the in-vivo situation in regards of tissue composition and cell configuration. While in-vivo animal models are a lot more lifelike, they are inherently connected with ethical concerns, translation to the human situation, logistic efforts and high costs. Here we explored the use of native human life slice cultures from explanted tibial plateaus upon their feasibility to serve as a highly lifelike and easy-to-handle model of the human joint which can be obtained in masses from few tissue samples.

Methods: Osteochondral slices $(1 \mathrm{~cm} \times 1 \mathrm{~cm} \times 500 \mu \mathrm{m})$ were prepared with a special microtome from 23 human tibial plateaus and subsequently cultivated in hanging inserts over the course of 7 or 21 days. Short-term cultivated slices were stimulated with either 800 pM/1.2 nM TNF-a or 800 pM TGF- $\beta 3$. During cultivation, viability of tissue cells was assessed via laser microscopy / resazurin assay. After cultivation, gene expression of cartilage ECM Marker proteins was quantified with RT-PCR. TNF-a stimulated slices and their controls were stained with Safranin-O and analyzed via histomorphometry to quantify tissue proteoglycan content.

Results: Tissue cell viability remained $>90 \%$ over the three weeks. Laser scanning microscopy revealed highly conserved spatial alignment of cells inside the cartilage. Incubation of the slice cultures with TNFa showed a significant, dose dependent decrease in mRNA expression of cartilage proteins collagen II, cartilage oligomeric matrix protein and aggrecan while incubation with TGF- $\beta 3$ caused a significant increase of early bone formation proteins collagen I and also cartilage oligomeric matrix protein. Histologically, TNF-a incubation caused a $32 \%$ reduction of proteoglycans, detachment of collagen fibers and cell swelling.

Conclusion: In summary, native osteochondral slice cultures provide a stable and manipulable, physiological model of human joint bone and cartilage biology. The setup is easy to produce and handle, scalable, and contributes to $3 \mathrm{R}$ principles in biomedical research, suggesting itself as a platform for preclinical testing especially regarding high-throughput applications.

\section{Background}

Musculoskeletal conditions account for a large amount of global disease burden (1) - especially diseases of the joint like osteoarthritis (OA), which keeps being the leading cause of disability worldwide. Due to rising prevalences of adiposity, modern inactive lifestyles and the increase in life expectancy, the relevance of osteoarthritis is constantly growing. Puig-Junoy et al. calculated, that the socioeconomic burden of $O A$ ranges between 0.25 and $0.50 \%$ of a country's gross domestic product (2). Until now, parts of joint physiology as well as the pathomechanism of OA are incompletely understood. Hence, there is no causal treatment for the underlying degeneration of the articular cartilage. For late stage OA, the most 
common strategy is the implantation of a total endoprosthesis. While to this day endoprosthetic replacement of the joint is the only viable option for end stage $O A$, the experience of the last decade with autologous chondrocyte transplantation showed the high capability of cartilage for regeneration - if the tissue is brought into a regenerative modus operandi (3). Hence, novel therapeutic approaches aim to regenerate the degraded cartilage tissue via cell therapy or stimulating molecules like Bone Morphogenic Proteins (4), (5). To accelerate this research, understanding of joint physiology, OA pathomechanisms and testing of novel therapeutic approaches is urgently needed.

Musculoskeletal research of the joint classically relies on distinct in-vitro and in-vivo approaches. In vitro models are based on cell lines (6) monolayer cultures (7) and cultures providing a three-dimensional (3D) cell surrounding (8) (9). While being the most economic and ethically supportable choice for investigation, all these models do not sufficiently represent the native situation. Even though tissue engineered cartilage models become more and more sophisticated, they mostly consist of primary cells which need to be cultivated in order to form new cartilage-like structures (10). These manipulations strongly affect the chondrocyte's metabolism and behavior. For example, the chondrocytes are not aligned in their typical spatial alignment and they lack the correct architecture and compositions of the cartilage extracellular matrix (ECM). Additionally, these models always only represent the cartilage tissue but not the adjacent bone - hence, the cellular crosstalk between the two compartments is insufficiently reflected. However, research has shown how important the interaction of the two tissues is - for example in the pathogenesis of $\mathrm{OA}(10)$.

In turn, animal models give the opportunity to perform experiments on the whole organism/intact tissue (11), (12). Besides being morally questionable, animal experiments are vastly time/cost-intensive to perform and to analyze. In advance, animals are no "smaller humans". While tissues are indeed often similar, one can never be certain that human tissue will react in the same way as the animal tissue does. Also, the strain on joints is different in humans (biped) and mice or cows (quadruped). Hence, a compromise is required, which allows for rapid and inexpensive in-vitro analysis without sacrificing too much of the physiologic tissue components. To develop such a model, we took advantage of the life slice technology known in neurophysiologic research which relies on the cutting and cultivation of thinnest tissue slices from most recently explanted human tissue (13). Osteochondral tissue explants have been described before and are topic of current organ modelling research - but so far, they mostly consist of animal osteochondral punches which have a diameter of at least $3 \mathrm{~mm}$ (14), (15), (16). Firstly, the diameter of this cultures makes sufficient nutrient supply by mere diffusion difficult. Even if the cells located in the deep tissue remain vital, perfusion of nutrients for an in-vivo-like cell metabolism (e.g. aminoacids for proteinsynthesis, co-factors for enzymes) is strongly restricted. Hence, cells are unlikely to react towards external stimuli as they would do in their native state. Some groups have tackled this challenge by the application of mechanical force to simulate the in-vivo mechanism of cartilage perfusion (16). This is a promising approach though it is not commercially available -making an easy adoption by other laboratories difficult. Another challenge with thick cartilage punches is their availability. With a 3-6 mm diameter, the amount of good quality punches that can be created of a human sample is very limited - making high-throughput applications difficult. So in order to create a model which provides 
wholesome perfusion combined with easy handling and obtaining, we aimed to adapt the successful concept of life slices from neurophysiological research to musculoskeletal research (13). This concept is based on cutting portions out of living tissue which are thin enough to be perfused and subsequently cultivate them long term to study native tissue conditions in-vitro. Therefore, we acquired 23 surgically explanted human tibial plateaus and created a total of 396 life slices. They were cultivated either for 7 or 21 days and evaluated upon their feasibility as a lifelike human joint model. The criteria for feasibility was ease of handling, long-term cell viability and adequate reaction to external stimuli. Therefore, the slices were cultivated in hanging inserts with- and without additional serum containing medium in order to assess the necessity of external stimuli to keep the cells alive and reactive. To assess the reactivity of cells inside the tissue, the slices were stimulated with known cytokines Tumor necrosis Factor a (TNF- $a$ ) and Transforming Growth Factor $\beta 3$ (TGF- $\beta 3$ ), which cause previously described cartilage alterations (17), (18). Reaction patterns were documented histologically with live tissue laser scanning confocal microscopy (CLSM) and Safranin-O staining, biochemically (Resazurin Assay) and on gene expression level (RNA isolation and qPCR).

\section{Methods}

\section{Sample acquisition and slice production}

Whole tibial plateaus (TPs; $n=23$ ) were acquired from patients undergoing endoprosthetic knee surgery during which TP were removed (ethical approval number EA4/072/18 - Charité - Universitätsmedizin Berlin). The specimens were directly transported in serum-free cell culture medium (Dulbeccos Modified Eagle Medium with $1 \mathrm{~g} / \mathrm{L}$ glucose; 1\% Penicilin/Streptomycin; 2\% HEPES, Merck, Darmstadt Germany) to the laboratory with a maximum delay of 1 hour. In the lab, TP were placed in petri dishes filled with prewarmed medium under a clean bench. After macroscopical inspection for an osteochondral site with well-preserved cartilage $v s$. bone relation, an orthopedic tissue punch (OATS ${ }^{\circledR}$, Arthrex, Naples, U.S.A.) press was used to create an osteochondral punch, $2 \mathrm{~cm}$ long and $10 \mathrm{~mm}$ in diameter (Fig. 1A). The punch was inserted into a 3D-printed microtome insert and subsequently cut into 24 individual slices per donor with diameters of 500-800 $\mu \mathrm{m}$ (Fig. 1B). Two slices were immediately conserved either in 5\% formaldehyde or RNAlater ${ }^{\circledR}$ (Thermofisher, Waltham U.S.A.). Slices in RNAlater were subsequently stored at $-80^{\circ} \mathrm{C}$. The remaining 22 slices were placed in a standing position into hanging inserts of 24-well plates with an $8 \mu \mathrm{m}$ pore diameter (Transwell ${ }^{\circledR}$, Corning, New York, U.S.A.) for optimal perfusion. All treatments of the tissue were performed with sterile surgical gloves and instruments (Fig. 1C).

Figure 1 - Production process of slice cultures. A. $2 \times 1 \times 1 \mathrm{~cm}$ long osteochondral cylinder is punched out of the macroscopically unaffected area of a recently explanted human tibial plateau under sterile conditions (time between surgery and preparation $<1 \mathrm{~h}$ ). B. The cylinder is inserted into a 3D-printed microtome insert with no additional fixation. $500 \mu \mathrm{m}$ thick slices are cut out from the cylinder. C. Slices are immediately transferred to a hanging insert of a multi-well plate and covered in cell culture medium.

\section{Maintenance and stimulation}


Slices of donors 1-6 were divided into a serum-free (F-) cultivation group and serum containing group $(\mathrm{F}+) . \mathrm{F}+$ group was cultivated in the same medium as mentioned above with an additional $10 \%$ fetal bovine serum (FBS). In both groups, 4 slices each were stimulated with either 300 pM TNF-a, 1.2 nM TNFa or 800 pM TGF-33 (Peprotech, USA) plus control group for either F+/F-. Stimulating factors were pipetted with low-binding tips (Corning, New York, U.S.A.) for minimal protein loss. Multiwell plates were cultivated at $37^{\circ} \mathrm{C} /$ in a humid $5 \% \mathrm{CO}_{2}$ atmosphere on a horizontal shaker going $10 \mathrm{rpm}$. Medium and stimulating factors were exchanged once after 3 days of culture. Slices of the additional 17 donors were cultivated without additional stimulating factors or FBS over the course of 21 days for a large group viability and gene expression analysis. Medium was completely exchanged twice a week.

\section{Viability analysis}

Briefly, a 10\% resazurin-medium solution (AlamarBlue ${ }^{\circledR}$, ThermoFisher Scientific, Waltham, U.S.A) was transferred into a 96-well plate $(300 \mu \mathrm{l} /$ well). The slices were taken out of their hanging insert, transferred into Resazurin-filled well and incubated for 2 hours at $37^{\circ} \mathrm{C} / 5 \% \mathrm{CO}_{2}$. Then, slices were taken out, placed back into their hanging inserts and fluorescence was measured with a plate reader (Synergy, BioTek, Winooski, U.SA.) applying an excitation wavelength of $540 \mathrm{~nm}$ and detection at $590 \mathrm{~nm}$. Viability of slices of Donor 1-6 was measured at day zero and day seven, donor 7-23 were measured at day 0, 7, 14 and 21 . In order to correlate results from the resazurin assay with an optical method, 13 Slices of two donors $(7 / 8)$ were cultivated for 3 consecutive weeks. On day 21, prior to the resazurin assay 2 slices per donor were heated to $60^{\circ} \mathrm{C}$ for 30 minutes and 2 slices for 1 hour in order to decrease intra-tissue cell viability to a minimum and have a portion of low-viability slices. Subsequently, a resazurin assay was performed on all slices. Afterwards, the slices were optically analyzed via live/dead staining with fluorescein diacetate (FDA, Sigma Aldrich, St. Louis U.S.A) and propidium iodide (PI, Sigma Aldrich, St. Louis U.S.A). For this purpose, slices were taken out of their hanging inserts, washed twice with Phosphate Buffered Saline (PBS) and incubated in $0.6 \%$ PBS-FDA (25 minutes), then in a 10\% PI solution (3 minutes). After a washing step, slices were immediately transferred into the microscopes incubating chamber. Tissue imaging was carried out with a Nikon Scanning Confocal $A 1 R s i+$ (Nikon, Tokyo, Japan) at $37^{\circ} \mathrm{C}$ in phenolred-free DMEM (Thermo Fisher Scientific, Waltham, USA). PI was excited at a wavelength of $488 \mathrm{~nm}$ and FDA at $485 \mathrm{~nm}$, respectively. The emission was detected at $590 \mathrm{~nm}$ (PI) and $514 \mathrm{~nm}$ (FDA). The scanning area was $2.82 \mathrm{~mm}^{2}$ and pictures were stitched with $20 \%$ overlap using the Nikon software (Nikon Capture NX-D, Vers. 1.6.2). Z-stacking was performed with $10 \mu \mathrm{m}$ spacing. Z-Stacks were rendered into a 3D-Image with living cells depicted in green and dead cells in red. The pictures were analyzed by using ImageJ (1.8.0; https://imagej.nih.gov/ij/plugins/cell-counter.html). Results show the sum of living cells per sample. For correlation, AlamarBlue ${ }^{\text {TM }}$ viability data was compared to the amount of living cells per $\mathrm{mm}^{3}$ sample. The picture volume was assessed by the Nikon software. Due to high standard deviations, each donor was tested individually using a two-tailed hyperbolic regression.

\section{RNA extraction and Quantitative RT-PCR}


For RNA isolation, cartilage was carefully dissected from bony tissue, slices were soaked with RNAlater (Thermofisher, Waltham, U.S.A.) and stored at $-80^{\circ} \mathrm{C}$ overnight. The next day, the frozen tissue was transferred into liquid nitrogen and pulverized with a special setup for very small tissue specimen (Biopulverizer 10-100 mg, BioSpec, Bartlesville, U.S.A.). Pulverized samples were transferred into TriReagent ${ }^{\circledR}-B C P($ Sigma-Aldrich, St. Louis, U.S.A.) solution followed by centrifugation for $45 \mathrm{~min}$ at $13000 \mathrm{~g}$. The aqueous phase was collected, and nucleic acids were precipitated by the addition of an equal volume of ice-cold isopropanol. After $30 \mathrm{~min}$ of incubation, precipitated nucleic acids were collected and resolved in RNA isolation buffer (RLT, Qiagen, Hilden Germany). Further purification was performed according to a protocol for animal tissues of the PicoPure ${ }^{\text {TM }}$ Kit (Thermofisher, Waltham, U.S.A.). Integrity and purity of RNA were analyzed using Agilent Bioanalyzer 2100 (Agilent, Palo Alto, USA) and a NanoDrop 1000 spectrophotometer (Thermo Fisher Scientific, Wilmington, USA) RNA was then reversely transcribed using a cDNA synthesis Kit (iScript ${ }^{\mathrm{TM}}$, BioRad, Hercules, U.S.A.). Real-time PCR was performed in triplicates in 96-well plates (Becton Dickinson, Franklin Lakes, U.S.A.) on a Mastercyclerep gradient realplex (Eppendorf, Hamburg, Germany) using expression assays for TaqMan probes and primer sets (orderno. in parentheses): collagen type I/ alpha 1(COL2A1, Ss03373344_g1), collagen type I alpha 1(COL1A1, Ss003373341_g1), aggrecan (ACAN, SS03373387_S1), Ki-67 (MKI67, qHsaCID0011882) and cartilage oligomeric matrix protein (COMP, Hs01572837_g1). In order to normalize the samples, the expression of SDHA (Succinate dehydrogenase complex, subunit A, Hs00188166_m1) was used. Marker gene expression is given as a percentage related to SDAH expression or as fold change compared to control samples applying the efficiency corrected $\Delta \Delta$-Ct method (19).

\section{Histology with Safranin-0}

Slices of each donor were fixated overnight in $5 \%$ formaldehyde solution and subsequently decalcified for 21 days in EDTA solution (Osteosoft ${ }^{\circledR}$, MERCK, Darmstadt, Germany). Afterwards, slices were frozen in liquid nitrogen and subsequently cut in a cryotome into $4 \mu \mathrm{m}$ thin slices. For Safranin-0 staining, slices were stained 30 minutes with $0.7 \%$ Safranin-O solution (Thermofisher, Waltham, U.S.A.). Counterstaining was performed with a $0.2 \%$ Fastgreen solution (Thermofisher, Waltham, U.S.A.) for one minute. To document ECM formation or loss, sections were mounted on glass slides; stainings were photodocumented using a light microscope (AX 10, Zeiss, Jena, Germany). The intensity of the Safranin$\mathrm{O}$ staining is directly proportional to the Glycosaminoglycan amount of the tissue and was therefore analyzed with a histomorphometric analysis as previously described (20). Briefly, pictures were taken and all pixels in the areas of interest were valued in the RGB colour mode with a tool based on Xcode (Apple, Sunnyvale, USA). When the red value (R) multiplied by 2 was higher than the sum of the green $(G)$ and blue (B) values, the pixel was counted as red. The intensity of each red pixel was calculated with this formula: intensity $=2 \times R$-value $-G$-value $-B$-value. Values of the intensity ranged between 1 and 508 . The mean intensity (sum of intensities/area of interest) was calculated from each image.

\section{Statistical Analysis.}


The significance level of log10-tranformed data was determined with the independent two sample t-test statistics of the Excel 2013 software package (Microsoft, Redmond, WA, USA). Normal distribution was checked applying the Anderson - Darling test, and equal variance of compared sample groups was tested applying the f-test. In all groups signals were normally distributed. If the equal variance test was not passed, Welch's t-test was applied. The dependence (correlation) was determined by calculating the Pearson correlation coefficient ( $r$ ) with Excel 2013 giving a value between +1 and -1 inclusive, where 1 is total positive correlation, 0 is no correlation, and -1 is total negative correlation. The distribution of the sample correlation coefficient (significance) was tested using Student's distribution. A p-value lower than 0.05 was considered as significant.

\section{Results}

To develop a physiological model for osteochondral tissue which allows for quick handling and highthroughput applications with limited human specimen at hand, we developed a method for the manufacturing of native osteochondral live slice cultures from human joint tissue. 500-800 $\mu \mathrm{m}$ thick osteochondral slice cultures were prepared from 23 different surgically explanted tibial plateaus with a special microtome insert and then cultivated in hanging inserts of 24-well plates (Fig. 1). In this study, we tested if the tissue cultures keep their physiological properties such as long-term cell viability, gene expression and responsiveness towards biological stimuli. In order to do so, tissue cultures of 6 donors were cultivated 7 days with additional stimulating cytokines and tissue cultures of 17 other donors were cultivated 21 days without any additional stimuli. Subsequently, the tissue cultures were analyzed microscopically, genetically and histologically.

Laser Microscopy shows highly conserved spatial cell order

In order to assess the viability and organization of cells in deep layers of cartilage tissue, culture slices were stained with PI/FDA and subsequently analyzed by CLSM. The amount of living cells was then compared to the results of the resazurin assay. Three-dimensional CLSM revealed $<10 \%$ chondrocyte viability in long-term heated slices, about $50 \%$ viability in short term heated slices and $>90 \%$ viability in non-heated slices (Fig. 2. A) after three weeks of prior cultivation time. Chondrocytes remained localized in their cartilage-typical, spatial alignment (column-like deep zone; pearl-bead structure in the mid zone; tightly packed cells in the superficial zone). No cell culture effects due to nutrition gradients were visible (e.g. no elongated cells in the peripheral tissue).

Resazurin assay correlates with visual assessed viability

To ensure that resazurin assay is sufficiently reflecting viability which might potentially be hampered by the dense ECM of osteochondral samples, selected samples of varying viability were analyzed with resazurin metabolic assay and CLSM. The results correlated with visually counted cells. A two-tailed Pearson correlation test showed a correlation ratio of $\mathrm{R}^{2}=80-84.5 \%$ of the metabolic assay with microscopical viability analysis (Fig. 2.C) which we regarded as sufficiently accurate to assess the general viability state of the tissue culture. Analysis of further tissue cultures was therefore performed 
with resazurin, since it allows for progredient viability analysis without the need of sacrificing the tissue slice with each measurement due to the toxic PI and hot laser beam of the CLSM.

Cell viability stays $<95 \%$ without additional serum over 21 days

After 7 days, F- cultivated slices of the cytokine stimulated groups exhibited a mean viability of $103 \pm$ $28.3 \%$ compared to day 0 with a mean standard deviation between the stimulation groups of $\pm 6 \%$. There was no significant difference between viability of day 0 and day 7 as well as between the four different cytokine stimulation groups. $\mathrm{F}+$ slices showed a significant increase in viability compared to day 0 . Viability increased to a mean of $220 \pm 60 \%$ with a mean standard deviation between the stimulation groups of $16.8 \%$ (Fig. 2. B). During the long-term cultivation, F- group showed viability of $114.24 \pm 43.35 \%$ after 7 days, $108.1 \pm 52.26 \%$ after 14 days and $113.37 \pm 55.61 \%$ after 21 days. Statistical analysis showed no significant differences in viability between the time points (Fig. 2. B).

Figure 2 - Cell viability analysis. A. cartilage viability analysis using CLSM. Pictures show three different slices from the same donor with Propidiumiodid(red)/Fluoresceindiacetat(green) staining. To induce cell death for correlation analysis, (from left to right) the first slice was heated 1 hours at $60^{\circ} \mathrm{C}$, the second slice was heated 30 minutes at $60^{\circ} \mathrm{C}$ and the third slice was left in its native state. Dead cells appear red, vital cells appear green. B. Metabolic viability analysis. Left chart shows mean viability on day seven of four groups stimulated with TNF-a (low 0.8 pM, high 1.2 pM) or TGF- $\beta 3$ (800 pM) after seven days with $(v)$-or without FBS $(\diamond)$-in relation to day $(n=6)$. Right chart depicts the cell viability in long-term cultivation after 7, 14 and 21 days; towards day 0, there was no statistically significant change detectable $(n=15)$. C. Correlation of visual and metabolic viability analysis. 13 slices of 2 different donors were analyzed microscopically and metabolically. For the individual donors, the two methods have a correlation value between 0.8 and 0.845 .

ECM protein transcription stays highly reactive to external stimulation in serum-free cultivation In order to test cell reactivity to external stimuli, we intended to induce visible changes in chondrocyte gene expression. Hence, slices were stimulated with TNF- $\alpha$ and TGF- $\beta 3$ to either suppress or induce cartilage relevant gene markers. Gene expression analysis showed different results in $\mathrm{F}$ - and $\mathrm{F}+$ stimulation group. In the F- group, TNF-a significantly reduced expression of COL2A1, ACAN and COMP. In relation to day 7 control, $C O L 2 A 1$ expression decreased to $11 \pm 7 \%$ at low dose TNF-a concentration and $5 \pm 5 \%$ at high-dose TNF-a. ACAN expression decreased to $42 \pm 33 \%$ in the lower concentration and $15 \pm$ $12 \%$ in the high concentration. COMP expression dropped to $44 \pm 48 \%$ in the lower concentration and to $10 \pm 14 \%$ in the high concentration. TGF- $\beta 3$ increased COMP expression 9-fold \pm 5 and COL $1 A 1$ expression by a mean foldchange of 326 - although with no statistical significance ( $\pm 434 ; p=0.15)$ (Fig. 3.A.). In the $\mathrm{F}+$ group, TNF-a decreased COL2A1 expression to only $33 \pm 35 \%$ in the lower concentration $(p=0.09)$ and to $11 \pm 16 \%$ in the high concentration towards day 7 negative control. Mean expression levels also decreased in $A C A N$ and $C O M P$ expression after TNF-a exposure, but the values exhibited lower significance than in the F- group ( $A C A N: \mathrm{p}=0.15$ and $\mathrm{p}=0.32$; COMP 0.08 and 0.20 ). TGF- $\beta 3$ significantly increased expression of COMP 29 -fold $( \pm 2.51 ; p=0.005)$ and mean COL $1 A 1$ expression to 6 -fold again with low statistical significance $( \pm 6.84 ; p=0.26)$ (Fig. 3.B.).

Effects on expression of proliferative markers are only distinguishable in serum-free cultivation 
Expression analysis of proliferation marker Ki-67 in F- cultivated groups upon TGF- $\beta 3$ stimulation showed an increase in expression levels after 7 days towards day 0 control by $3738 \pm 5987 \%$, though not statistically significant $(p=0.06)$. In the $\mathrm{F}+$ group, however, the negative control of day 7 and all 3 stimulation groups exhibited statistically significant changes towards day 0 . Mean increase of expression of all 4 groups resulted in a $5.19 \times 10^{5} \pm 5.9 \times 10^{5}$ fold change $(p=0.003)$. No statistically significant differences were detected between the different $F+$ cultivated stimulation groups in relation to day 7 negative control (Fig. 3. A. and B.).

Expression of essential cartilage ECM ACAN and COL2A1 proteins stays stable for about 14 days in unstimulated slice culture

Expression of cartilage relevant genes COL2A1 and $A C A N$ stays stable for 14 days and then starts to significantly decline on day 21 (COL2A1: $32 \pm 23 \%$; and ACAN $51 \pm 64 \%$ of day 0) (Fig. 3.C.). Cartilage remodeling marker $C O L 1 A 1$ shows a short increase after 7 days but then returns to levels of day 0 at day 21. Expression of $C O M P$ reacted very promptly to the cultivating situation with a significant drop in expression levels to $45 \pm 30 \%$ after 7 days to $14 \pm 11 \%$ after 21 days, respectively. Expression of Ki- 67 as a marker for cell proliferation increases up to $100.000 \%$ overtime in relation to day 0 but the increase is inignificant and the high relative increase at the measurement points are a result of the very low absolute initial expression of $\mathrm{Ki}-67$ at day 0.

Figure 3 - Gene expression analysis A. RT-PCR of stimulated F- cultivated slices. Bar charts show the quantitative gene expression of osteochondral slices stimulated with either low dose TNF-a (light grey), high dose TNF- a (dark grey) or TGF- $\beta 3$ (black) stimulated osteochondral slices in F- medium. Shown are cartilage relevant markers gene COL2A1, COL1A1, ACAN, COMP and the proliferation relevant gene Ki-67 after 7 days of cultivation in comparison to the negative control with Succinate dehydrogenase complex subunit $A$ as reference gene. Scales are logarithmic for clear presentability ( $n=6$ donors). B. RT-PCR of stimulated F + cultivated slices. Graphs show the same setup as the graphs in A but with $10 \% \mathrm{FBS}$ addition $(n=6)$. C. RT-PCR of long-term cultivated slice cultures. Gene expression of 21 days cultivated slices at day 7,14 and 21 in relation to day 0 ( $n=12$ donors).

Tissue slices react to TNF- $a$ with visible remodeling of ECM and cellular swelling To analyze, whether the native slice cultures preserve their tissue-specific functionality to remodel extracellular matrix, slices were stained with Safranin-O. Proteoglycan content of TNF-a stimulated slices and negative controls was quantified via histomorphometry (Fig. 4. A). In accordance to the alterations in gene expression, TNF-a stimulated slices showed strongly altered histomorphology. Intra-cartilage fibers appeared less dense and chondrocyte increased in volume and diameter (Fig. 4. B). Proteoglycan content in the TNF-a-treated group was also significantly reduced: Intensity of Safranin-O staining declined by $32 \% \pm 24(p=0.04)$ compared to the untreated controls (Fig. 4. C).

Figure 4 - Histological analysis ( $\mathrm{n}=6$ donors) A. Histomorphometrical analysis - Histomorphometrical analysis of Safranin-O stainings of all experimental groups considering the proportion and intensity of the stained area as the mean intensity normalized to the control + standard deviation. B. Representative 
picture of TNF-treated slice and control. TNF-a-treated slices show less intense Safranin-O staining, loosened matrix structure and swollen cells in the mid zone of the cartilage. C. Comparison of color intensity in stimulated and non-stimulated slices. TNF-a stimulated slices show a mean reduction of redintensity of $32 \%(n=6)$.

\section{Discussion}

Osteochondral live slice cultures uphold high viability, spatial cell alignment and ECM gene expression Despite their thin preparation, osteochondral live slice cultures conserve high cell viability and tissue specific behavior under easy-to-handle cultivation conditions. CLSM revealed high cell viability even in deeper tissue levels. Hence, the slice thickness of $500 \mu \mathrm{m}$ seems to be optimal for cell protection within tissue and sufficient nutrient perfusion by movement-enhanced diffusion. Since chondrocytes are of mesenchymal origin, it might be possible, that they leave their tissue specific position and migrate to the periphery of the tissue, but in our setting CLSM showed highly conserved cell architecture inside the tissue (21). Cartilage is divided into three different cellular zones: The superficial zone (tightly packed with MSC like cells), the mid zone (pearl-bead-like alignment) and the deep zone (column-like chondrocyte alignment) (22) (23). The conservation of the cellular zones depends on the supply with nutrients and physiological cartilage infrastructure - minimizing cellular stress by malnutrition and/or hypoxia (24).

The cell viability assessed via CLSM highly correlated with the results of the resazurin assay - making the resazurin assay applicable for accurate viability analysis. We consider this an important finding, since CLSM viability analysis is time-consuming and has low availability. A high throughput model, however, should be fast and easy to handle. Furthermore, the resazurin assay is a completely non-toxic procedure which allows for toxicity analysis including multiple measurements - while CLSM always damages the tissue due the hot laser beam and therefore can only be used for end point analysis. The short-term cultivation (7 days) showed that while the F-cultivated slices showed very little variation in viability, addition of $\mathrm{F}+$ cultivated slices increased viability readouts by about $220 \%$ - most likely due to increase of metabolic turn-over by stimulating serum proteins. Hence, we assume that serum addition to the cultures could possibly occlude cell toxic effects by externally applied substances under investigation by the user. Nevertheless, the subsequent long-term cultivation without any additional stimuli showed highly stable viability over the course of 21 days. These advantages suggest this method applicable for a variety of research purposes aiming to analyze specific intra-cartilage processes over a longer time period without the necessity of serum addition to keep the cells alive. Gene expression of cartilage relevant ECM marker genes like collagen II and aggrecan stayed relatively stable for 14 days before abruptly decreasing. Similar genetic dynamics have been observed beforehand in explant culture models which used immobilized, 6-mm-diameter bovine cartilage discs (25). These results indicate, that osteochondral live slices do not additional treatment like immobilization in agarose or strong perfusion pressures for nutrition and retaining their tissue specific genetic phenotype - effectively reducing the handling necessities by a great deal.

Tissue reacts quickly to biological stimuli and stays vibrant for active remodeling 
Stimulation of the osteochondral slices showed that not only the histomorphology remained unchanged but also that the cells properly reacted to external stimulation. TNF-a is a pro-inflammatory cytokine and well known for the induction of cartilage matrix degradation - both in-vivo and in-vitro (26) (27). However, Pretzel et al. required 14 days to achieve minimal proteoglycan reduction with TNF-a mono treatment in a $3 \mathrm{~mm}$ diameter porcine explant model; other models required the addition of other potent degrading proteins such as Oncostatin-M or Interleukin-1 $\beta$ to reach similar results (14), (28), (29). In the F-cultivated group, addition of TNF-a to the slices showed a dose dependent decrease in expression cartilage ECM relevant genes $C O L 2 A 1, A C A N$ and $C O M P$ and an active reduction of proteoglycans in the tissue by $32 \%$ in only 7 days. TGF- $\beta 3$ addition resulted in highly increased expression of COMPand Ki-67. Additional to visible cellular alterations, histomorphometrical analysis revealed a reduction of proteoglycans and loosening of cartilage matrix by TNF-a stimulation. These changes do not only require the reduced production of matrix proteins, but also the TNF-a induced, active production of matrix-degrading enzymes like Matrixmetalloproteinase - 1 (MMP-1), MMP-2, MMP-13, MMP-14 and others (30), (31) (32). This suggests that even the cells in the deepest tissue layers retain the reactivity towards their tissue and are sufficiently nurtured for production and secretion of enzymes.

In the $\mathrm{F}+$ group however, only the high dose TNF-a dependent reduction of COL2A1 and TGF- $\beta 3$ dependent increase of $C O M P$ reached statistical significance towards their control at day 7. Analysis of $K i-67$ showed that while all stimulation subgroups within the $F+$ group including the negative control rose enormously at the F-group only TGF- $\beta 3$ resulted in higher expression. This further supports the thesis, that serum addition causes strong and unspecific stimulations, which occlude the specific effects of other stimuli. This gives us the impression, that serum addition is not only non-essential for the survival of the chondrocytes but also causes undesired artifacts in the analysis of cell reaction. For the purpose of toxicity screening, the redundancy of serum addition is very desirable since serum is a highly inconsistent protein mixture and an unknown variable in every experimental setup - especially those of pharmacologic nature.

\section{Conclusion}

Native human osteochondral life slices pose a highly lifelike model for musculoskeletal research and high-throughput screening of tissue reactions in their native state, which can be obtained in bulk from just a single human sample. Despite of low effort manufacturing, the tissue completely retains its microarchitecture and the cells conserve their tissue specific phenotype as well as their ability to properly react towards different stimuli. This native tissue culture combines the cost effectiveness/low logistic effort of 2-D cell cultures and the native tissue state of animal models. The easy production and thin nature of the slices make it possible to create up to 50-100 pieces from just one specimen. Combined with the easy cultivation in hanging inserts effectively enables high throughput screening of threedimensional tissue processes - which would otherwise take weeks of preparation when performed in a tissue engineered 3D culture or even in an animal model. Additionally, this tissue culture makes the use of serum for cell expansion/survival obsolete thereby eliminating the addition of an undefined protein mixture, probably causing unwanted effect in the experiments. In summary, the native life-slice culture is 
suitable as a tissue model for answering numerous scientific questions in the musculoskeletal research especially for dosage and toxicity studies which are normally conducted in-vivo. It brings us a step closer to 3R: more thorough research with less necessity for animal experiments.

\section{Abbreviations}

$\cdot \mathrm{OA}$

- Osteoarthritis

- ECM

- extracellular Matrix

- FBS

- fetal bovine serum

- TP

- Tibial plateau

- F-

- serum-free

- F+

- serum containing

- PBS

- Phosphate Buffered Saline

- FDA

- Fluourescein diacetate

- PI

- Propiudium iodid

- CLSM

- confocal laser scanning microscopy

- TNF-a

- Tumor necrosis Factora

- TGF-B3

- Transforming Growth Factor $\beta 3$

- MMP

- Matrixmetalloproteinase

\section{Declarations}

Ethics approval and consent to participate:

Study was approved by ethics committee of Charité - Universityhospital Berlin, Campus Benjamin Franklin (Internal Review Number EA4/072/18). Consent for tissue use was acquired by every patient individually at least 2 days before surgery. 


\section{Consent for publication}

Not applicable

\section{Availability of data and materials}

The datasets supporting the conclusions of this article are included in the supplemental information.

\section{Competing interests}

The authors declare that they have no competing interests

\section{Funding}

This research project was mainly funded by the Einstein Centre for regenerative Therapies. The EinsteinFoundation funds research in regenerative medicine with a focus on highly translational approaches and pursues no commercial interests in its funding.

\section{Authors' contributions}

JS was involved in the conception, design, data acquisition and analysis as well as manuscript writing. MS and TD were mainly involved in study design- and conception and TD performed the computerized histomorphometrical analysis. HM and AAK were involved in the study design- and conception as well as histologic preparation of tissue sections. US, DK and CR were involved in patient recruitment, tissue acquisition and preparation. All authors were involved in data interpretation and manuscript drafting, reviewing, and development. The views and opinions expressed within this manuscript are those of all authors. All authors read and approved the final manuscript.

\section{Acknowledgements}

We thank our colleagues from Charité Research Workshop for crafting special instruments for the slice culture manufacturing. We thank Christoph Müller and Anja Fleischmann for assistance with tissue culture maintenance. We would also like to show our gratitude to the core unit cell harvest for organizing the logistics of the life tissue transport.

\section{Authors' information}

JS is MD/PhD candidate at Charité - Univsersityhospital Berlin (CUB). MS is head of laboratory for tissue engineering at CUB. TD is senior postdoc at laboratory for tissue engineering at CUB. AK is head of coreunit iPATH for specialized histopathology at CUB. HM is senior postdoc at DRFZ, Berlin. DK is head of the department of Orthopedics at Helios clinic, Berlin-Buch. US is chief resident at the department of orthopedics (section of knee-endoprosthetics) at CUB. CR is chief resident at the department of maxillofacial surgery at CUB. 


\section{References}

1. CK, Vina ER and Kwoh. Epidemiology of Osteoarthritis: Literature Update. Curr Opin Rheumatol. 30(2): 160-167. doi: 10.1097/BOR.0000000000000479, 2018.

2. Puig-Junoy J, Zamora Becon AR. Socio-economic costs of osteoarthritis: A systematic review of cost-of-illness studies. Semin Arthritis Rheum Apr. 2015;44(5):531-41. doi:

10.1016/j.semarthrit.2014.10.012 .

3. CB. Foldager. Advances in autologous chondrocyte implantation and related techniques for cartilage repair. Dan Med J Apr. 2013;60(4):B4600.

4. Lu CH, Yeh TS, Yeh CL, Fang YH, Sung LY, Hu YC. et al. Regenerating cartilages by engineered ASCs: prolonged TGF- 33 /BMP- 6 expression improved articular cartilage formation and restored zonal structure. Mol Ther. 2014;22(1):186-95. doi:10.1038/mt.2013.165.

5. Deng ZH, Li YS, Lei GH, Huard J. Bone morphogenetic proteins for articular cartilage regeneration. Osteoarthritis Cartilage Sep. 2018;26(9):1153-61. doi: 10.1016/j.joca.2018.03.007 .

6. chadow S, Siebert HC, Lochnit G, Kordelle J, Rickert M, Steinmeyer J. Collagen metabolism of human osteoarthritic articularcartilage as modulated by bovine collagen hydrolysates. PLoS One. 2013;8:e53955.

7. Stradner MH, Gruber G, Angerer H, Huber V, Setznagl D,Kremser, M L, Furst F. C., Windhager R, Graninger W B. Sphingosine-1-phosphate counteracts the effects of interleukin-1beta inhuman chondrocytes. Arthritis Rheum. 2013, 65(8), 2113 - 2122.

8. Schlichting N, Dehne T, Mans K, Endres M, Stuhlmüller B. et al. Suitability of porcine chondrocyte micromass culture to model osteoarthritis in vitro. Mol Pharm. 2014 Jul 7;11(7):2092-105.

9. De Moor L, Beyls E, Declercq H Scaffold Free Microtissue Formation for Enhanced Cartilage Repair. Ann Biomed Eng 2019, doi:10.1007/s10439-019-02348-4.

10. Sharma AR, Jagga S, Lee SS, Nam J. nterplay between cartilage and subchon-dral bone contributing to pathogenesis of osteoarthritis. Int J Mol Sci. 2013;14:19805-30. doi:10.3390/ijms141019805.

11. Jimenez PA, Glasson SS, Trubetskoy OV, Haimes HB. Spontaneous osteoarthritis in Dunkin Hartley guinea pigs: histologic, radiologic, and biochemical changes. Lab Anim Sci. 1997;Dec;47(6):598601.

12. Kuyinu E, Narayanan G, Nair LS, Laurencin CT. Animal models of osteoarthritis: classification, update, and measurement of outcomes. J Orthop Surg Res. 2016;11:19.

13. Schwarz N, Uysal B, Welzer M, Bahr JC, Layer N, Koch H. et al. Long-term adult human brain slice cultures as a model system to study human CNS circuitry and disease. eLife. 2019;8:e48417. doi:10.7554/eLife.48417.

14. Pretzel D, Pohlers D, Weinert S, Kinne RW. In vitro model for the analysis of synovial fibroblastmediated degradation of intact cartilage. Arthri Res Ther. 2009;11:R25.

15. Haltmeyer E, Ribitsch I, Gabner S, Rosser J, Gueltekin S, Jenner F. et al. Co-culture of osteochondral explants and synovial membrane as in vitro model for osteoarthritis. PLoS One. 
2019;14(4):e0214709.

16. M.L.Vainieriab, D.WahlaM, AliniaG.J.V.M, van Oschb, S.Grada. Mechanically stimulated osteochondral organ culture for evaluation of biomaterials in cartilage repair studie. Acta Biomaterialia. 81 Pages 256-266, 2018.

17. Hu G, Zhao X, Wang C, Geng Y, Zhao J, Zhang X. et al. MicroRNA-145 attenuates TNF-a-driven cartilage matrix degradation in osteoarthritis via direct suppression of MKK4. Cell Death Dis Oct 26;8(10) doi:10.1038/cddis.2017.522., 2017.

18. Finnson K, Chi $>$, Bou-Gharios G, Leask A and Philip A. TGF-b signaling in cartilage homeostasis and osteoarthritis. Front Biosci (Schol Ed). Jan 1;4:251 - 68., 2012.

19. MW, Pfaffl. A new mathematical model for relative quantification in real-time RT-PCR. Nucleic Acids Res. 29 (45), 2001.

20. Schlichting N, Dehne T, Mans K, Endres M, Stuhlmüller B, Sittinger M, Kaps C, Ringe J. Suitability of porcine chondrocyte micromass culture to model osteoarthritis in vitro. Mol Pharm. 2014;11:2092105. doi:10.1021/mp5000554.

21. Hopper N, Henson F, Brooks R, Ali E. Rushton N, and Wardale J. Peripheral blood derived mononuclear cells enhance osteoarthritic human chondrocyte migration. Arthritis Res Ther. 2015;17(1):199. doi:10.1186/s13075-015-0709-z.

22. Karim A, Amin A, Hall A. The clustering and morphology of chondrocytes in normal and mildly degenerate human femoral head cartilage studied by confocal laser scanning microscopy. J Anat Apr. 2018;232(4):686-98. doi:10.1111/joa.12768. J Anat.

23. Takada S. E and Mizuno. Reproduction of Characteristics of Extracellular Matrices in Specific Longitudinal Depth Zone Cartilage within Spherical Organoids in Response to Changes in Osmotic Pressure. Int J Mol Sci. 2018 May; 19(5): 1507. doi: 10.3390/ijms19051507.

24. Youn I, Choi JB, Cao L. Setton LA and Guilak F. Zonal variations in the three-dimensional morphology of the chondron measured in situ using confocal microscopy. Osteoarthritis Cartilage Sep. 2006;14(9):889-97. doi:10.1016/j.joca.2006.02.017.

25. Moo EK. Abu Osman NA and Pingguan-Murphy B. The metabolic dynamics of cartilage explants over a long-term culture period. Clinics Aug. 2011;66(8):1431-6.

26. Manicourt DH, Poilvache P. Van Egeren A, Devogelaer JP, Lenz ME and Thonar EJ. Synovial fluid levels of tumor necrosis factor alpha and oncostatin $\mathrm{M}$ correlate with levels of markers of the degradation of crosslinked collagen and cartilage aggrecan in rheumatoid arthritis but not in osteoarthritis. Arthritis Rheum Feb;43(2):281-8. doi:10.1002/1529-0131, 2000.

27. Hui W, Rowan AD. Richards CD and Cawston TE. Oncostatin M in combination with tumor necrosis factor alpha induces cartilage damage and matrix metalloproteinase expression in vitro and in vivo. Arthritis Rheum Dec. 2003;48(12):3404-18. doi:10.1002/art.11333.

28. Thudium CS, Engstrom A, Groen S, Karsdal M, Bay-Jensen AC. An Ex Vivo Tissue Culture Model of Cartilage Remodeling in Bovine Knee Explants. J Vis Exp 3;(153). doi:10.3791/59467., 2019. 
29. Clutterbuck A, Mobasheri A, Shakibaei M, Allaway D, Harris P. Interleukin-1beta-induced extracellular matrix degradation and glycosaminoglycan release is inhibited by curcumin in an explant model of cartilage inflammation. Ann N Y Acad Sci. 2009;1171:428-35. doi:10.1111/j.17496632.2009.04687.x.

30. Yoon SW, Chun JS, Sung MH, Kim JY, Poo H. a-MSH inhibits TNF-a-induced matrix metalloproteinase-13 expression by modulating p38 kinase and nuclear factor $\mathrm{KB}$ signaling in human chondrosarcoma HTB-94 cells. Osteoarthritis Cartilage 16(1) doi:10.1016/j.joca.2007.05.02, 2008.

31. Kelley M, Rose A, Song K, Chen Y, Bradley J, Acott TS. et al. Synergism of TNF and IL-1 in the Induction of Matrix Metalloproteinase-3 in Trabecular Meshwork. Glaucoma 48(6) doi:10.1167/iovs.06-1445, 2007.

32. $10.1186 / 1423-0127-21-12$

Tsai CL, Chen WC, Hsieh HL. Chi PL Yang CM et al. TNF-a induces matrix metalloproteinase-9dependent soluble intercellular adhesion molecule-1 release via TRAF2-mediated MAPKs and NF-KB activation in osteoblast-like MC3T3-E1 cells. J Biomed Sci. 5;21(1):12 doi: 10.1186/1423-0127-2112., 2014.

33. Schwarz N, Uysal B, Welzer M, Bahr JC, Layer N, Löffler H. et al. Long-term adult human brain slice cultures as a model system to study human CNS circuitry and disease. eLife. 2019;8:e48417.

\section{Figures}




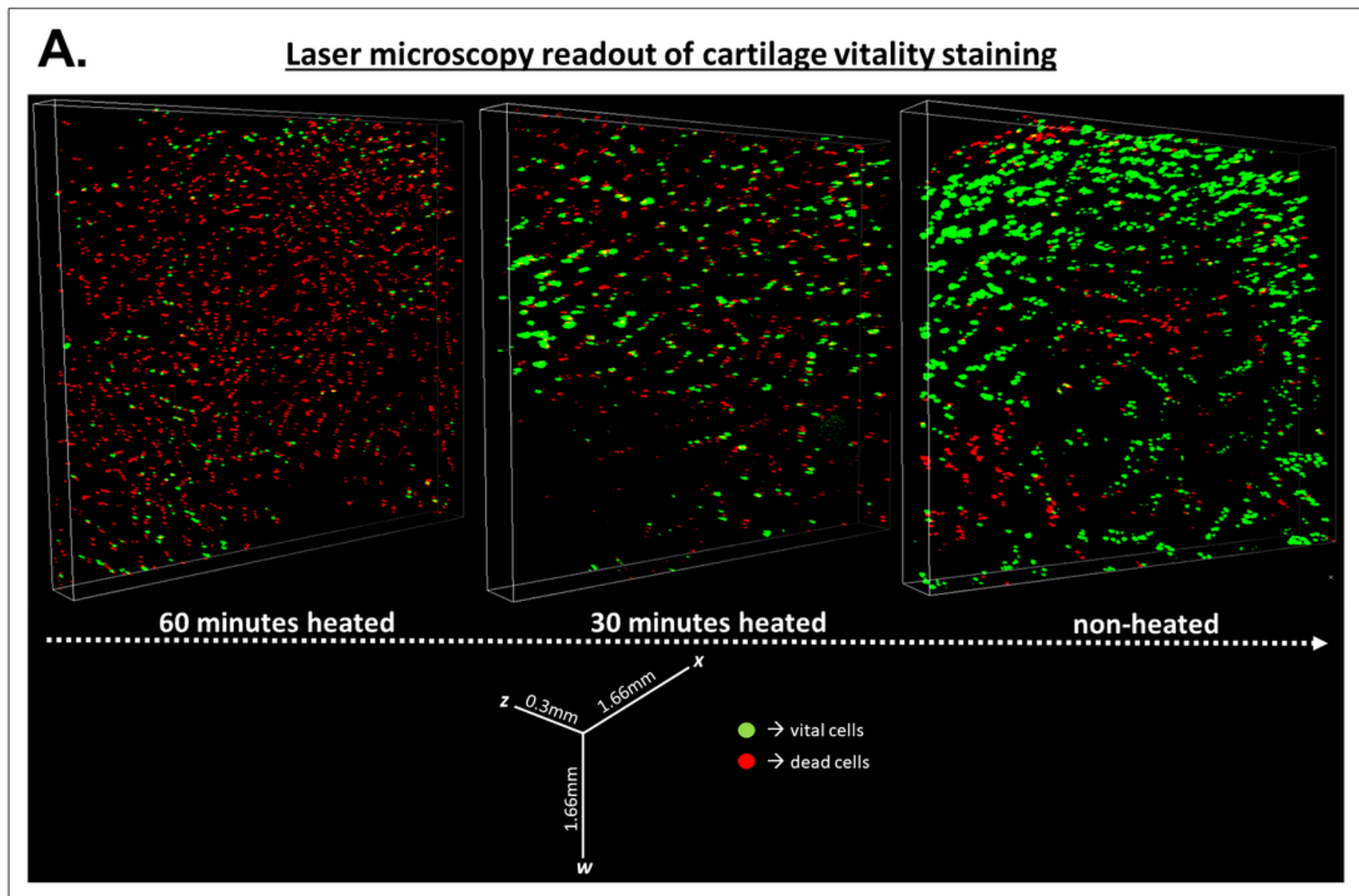

B.

Influence of serum on viability

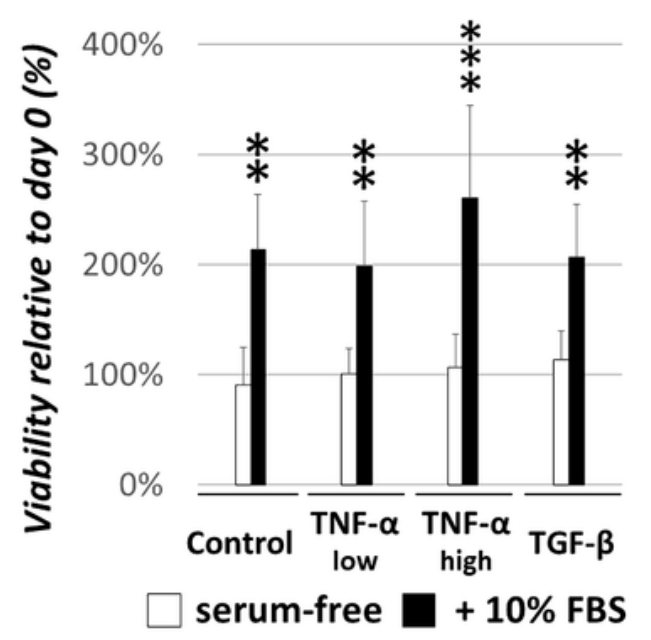

\section{Viability over 21 days} $400 \%$

$300 \%$

$200 \%$

$100 \%$

$0 \%$

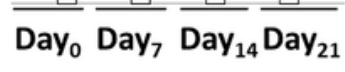

C. Correlation of vitality/viability $m^{m} 5000 \quad R_{\Delta}{ }^{2}=0.845$

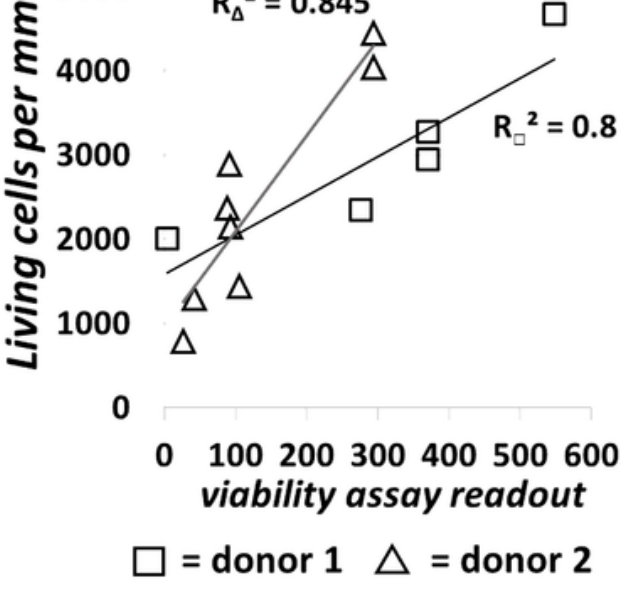

Figure 1

Production process of slice cultures. A. $2 \times 1 \times 1 \mathrm{~cm}$ long osteochondral cylinder is punched out of the macroscopically unaffected area of a recently explanted human tibial plateau under sterile conditions (time between surgery and prepara-tion $<1 \mathrm{~h}$ ). B. The cylinder is inserted into a 3D-printed microtome

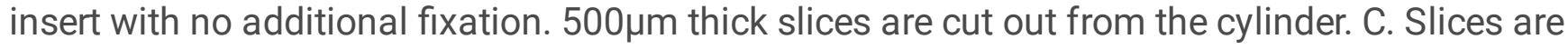
immediately transferred to a hanging insert of a multi-well plate and covered in cell culture medium. 


\section{A. Unstimulated control}

Safranin-O Staining Histomorphometric analysis

Donor 1.

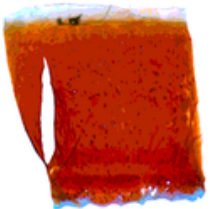

Donor 2.
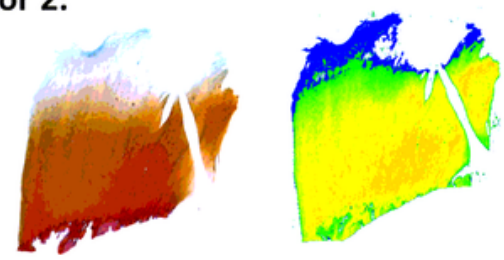

Donor 3.
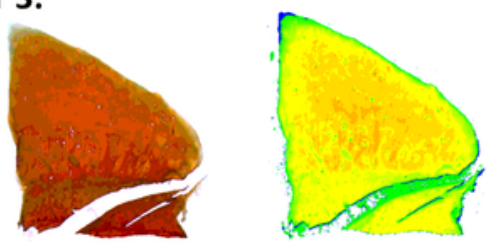

\section{TNF- $\alpha$ stimulated slices}

Safranin-O Staining Histomorphometric analysis

Donor 1.
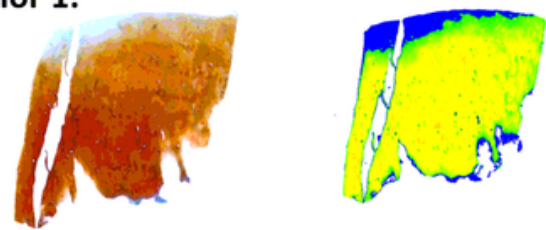

Donor 2.
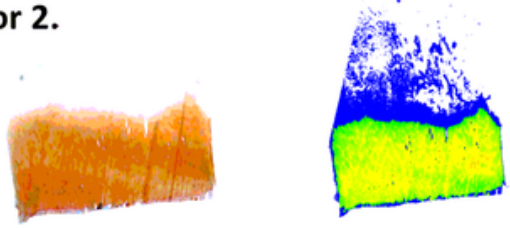

Donor 3.

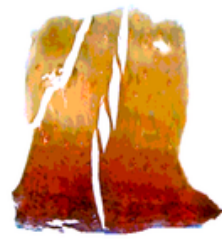

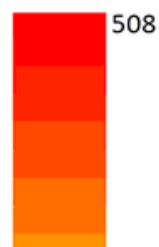

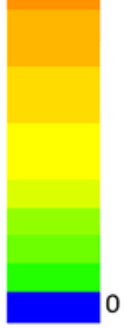

Colour intensity scale
B. Comparison in cartilage morphology
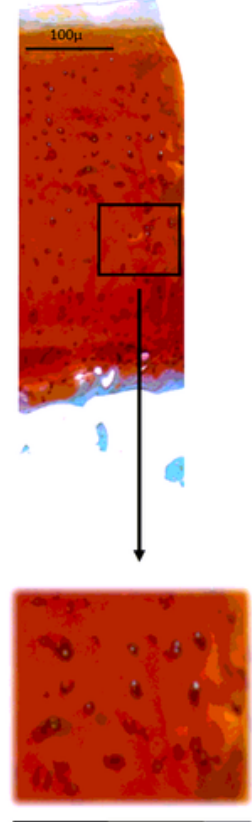

Negative control
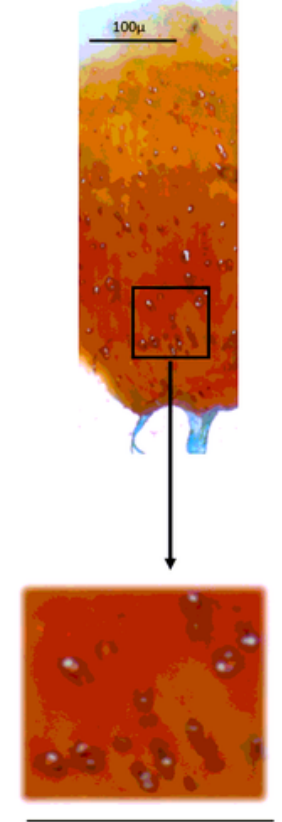

1,2 nM TNF- $\alpha$
C. Reduction of Proteoglycan content in TNF- $\alpha$ stimulated slices

100

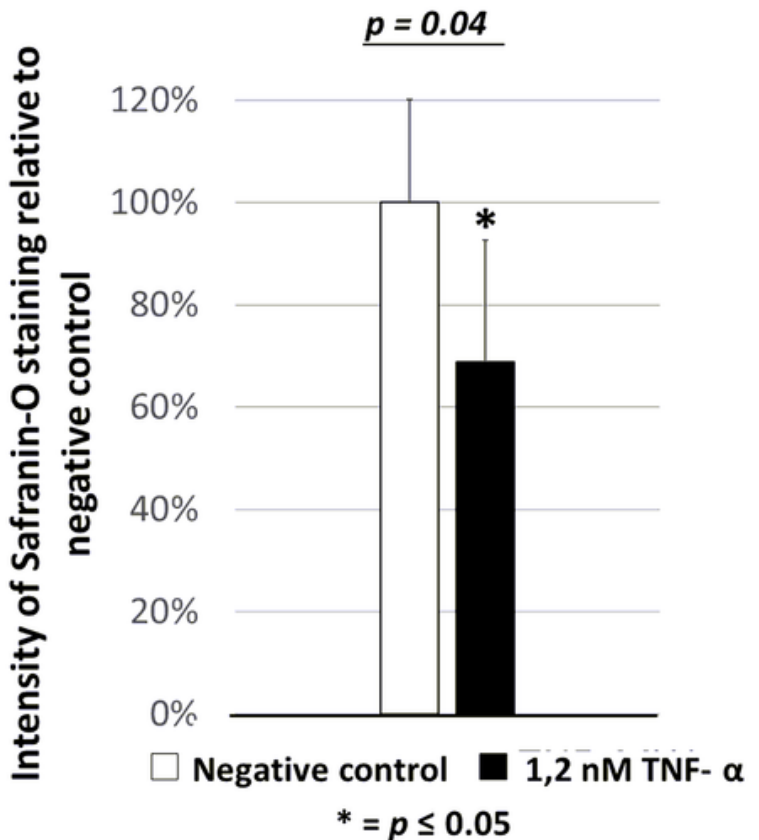

Figure 2

Cell viability analysis. A. cartilage viability analysis using CLSM. Pictures show three different slices from the same donor with Propidiumiodid(red)/Fluoresceindiacetat(green) staining. To induce cell death for correlation analysis, (from left to right) the first slice was heated 1 hours at $60^{\circ} \mathrm{C}$, the second slice was heated 30 minutes at $60^{\circ} \mathrm{C}$ and the third slice was left in its native state. Dead cells appear red, vital cells appear green. B. Metabolic viability analysis. Left chart shows mean viability on day seven of four groups 
stimulated with TNF-a (low 0.8 pM, high 1.2 pM) or TGF- $\beta 3$ (800 pM) after seven days with( () )- or without FBS ( () -in relation to day $(n=6)$. Right chart depicts the cell viability in long-term cultiva-tion after 7,14 and 21 days; towards day 0 , there was no statistically significant change detectable $(n=15)$. C. Correlation of visual and metabolic viability analysis. 13 slices of 2 different donors were analyzed microscopically and metabolically. For the individual donors, the two methods have a correlation value between 0.8 and 0.845 .
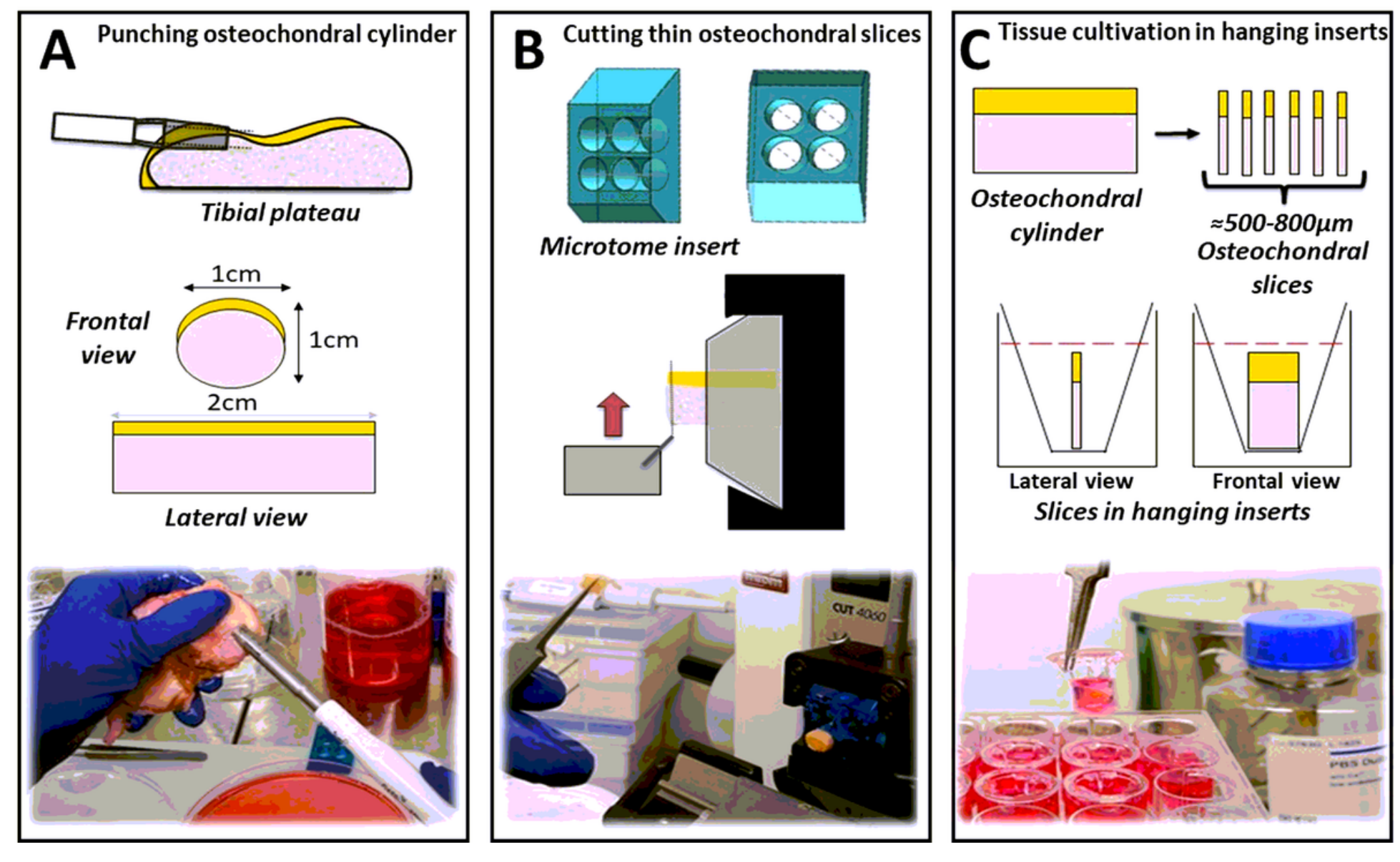

Figure 3

Gene expression analysis A. RT-PCR of stimulated F- cultivated slices. Bar charts show the quantitative gene expression of osteochondral slices stimulated with either low dose TNF-a (light grey), high dose TNF- $a$ (dark grey) or TGF- $\beta 3$ (black) stimulated osteochondral slices in F- medium. Shown are cartilage relevant markers gene COL2A1, COL1A1, ACAN, COMP and the proliferation relevant gene Ki-67 after 7 days of cultivation in comparison to the negative control with Succinate dehydrogenase complex subunit A as reference gene. Scales are logarithmic for clear presentability ( $n=6$ donors). B. RT-PCR of stimulated F+ cultivated slices. Graphs show the same setup as the graphs in A but with $10 \%$ FBS addition $(n=6)$. C. RT-PCR of long-term cultivated slice cultures. Gene expression of 21 days cultivated slices at day 7, 14 and 21 in relation to day 0 ( $n=12$ donors). 
Shortterm cultivation

Serum-free cultivation

A.

$10 \%$ FBS cultivation

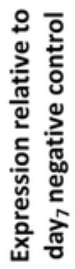

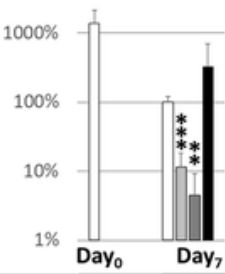

B.

COL2A1

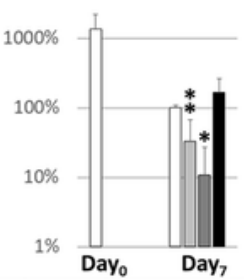

COL1A1
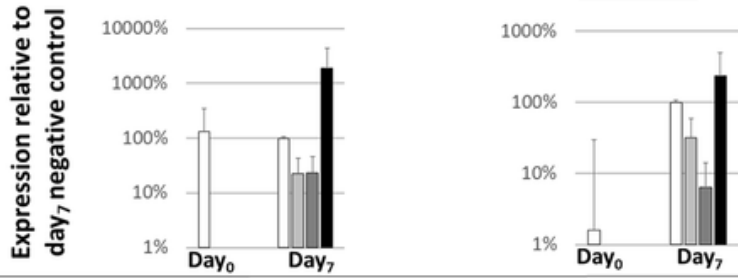

ACAN

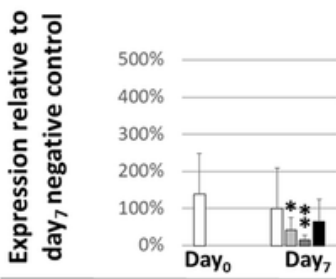

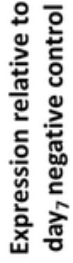

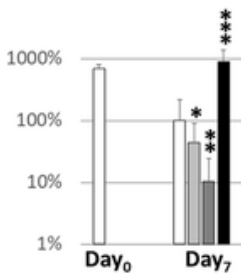

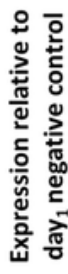

$10000000 \%$

$100000 \%$

$1000 \%$
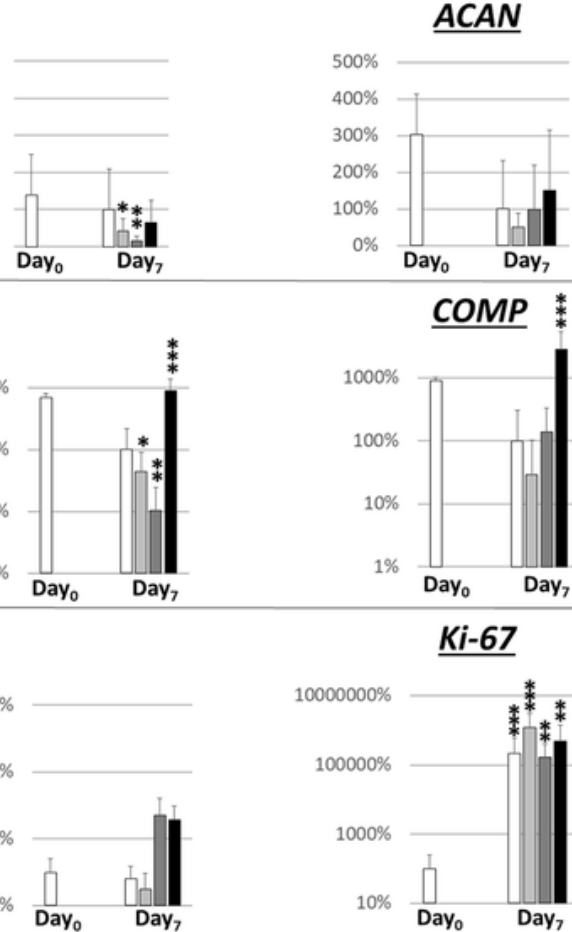

COMP $*$

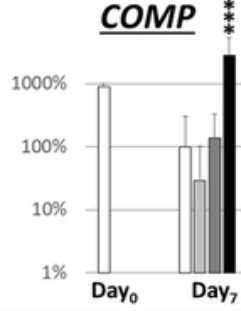

$\underline{K i-67}$

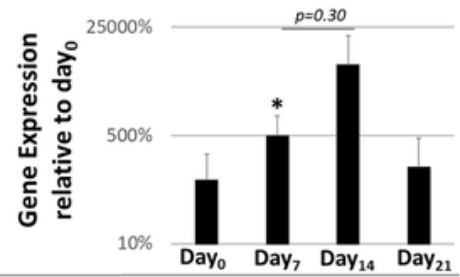

\section{Serum-free cultivation}

Longterm cultivation

C.
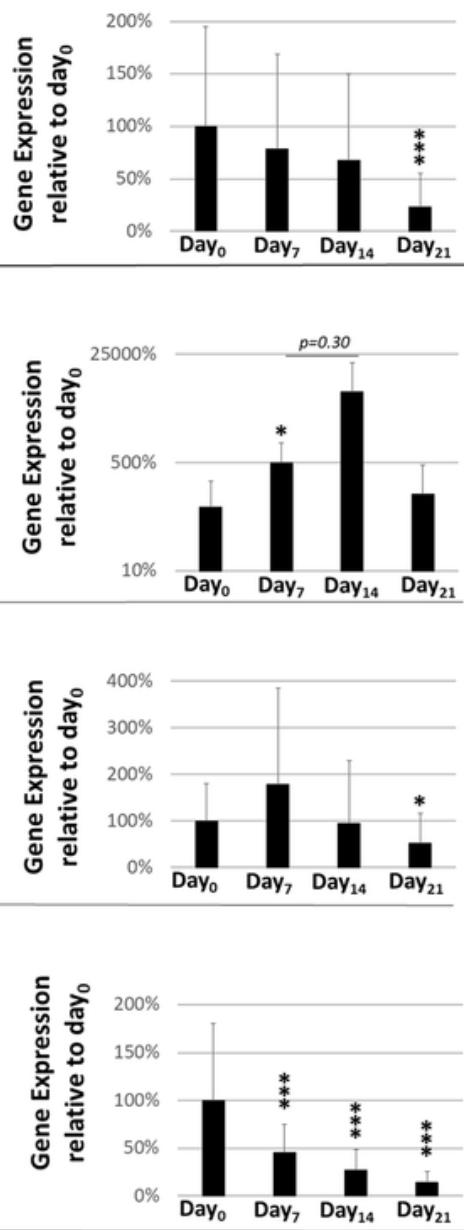

$\square$ Control

$\square$ TNF- $\alpha 300 \mathrm{pM}$

$\square$ TNF- $\alpha 1.2 \mathrm{nM}$

- TGF- $\beta 3800$ pM

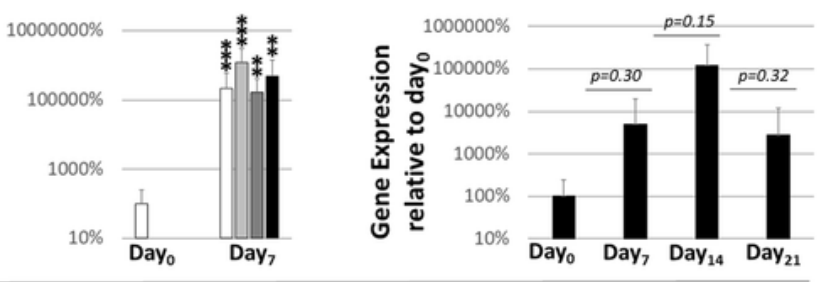

$*=P \leq 0.05$

$*=p \leq 0.01$

$\underset{*}{*}=p \leq 0.001$

Figure 4

Histological analysis ( $n=6$ donors) A. Histomorphometrical analysis - Histomorphometrical analysis of Safranin-0 stain-ings of all experimental groups considering the proportion and intensity of the stained area as the mean intensity normal-ized to the control + standard deviation. B. Representative picture of TNF-treated slice and control. TNF-a-treated slices show less intense Safranin-O staining, loosened matrix 
structure and swollen cells in the mid zone of the cartilage. C. Comparison of color intensity in stimulated and non-stimulated slices. TNF-a stimulated slices show a mean reduction of red-intensity of $32 \%(n=6)$.

\section{Supplementary Files}

This is a list of supplementary files associated with this preprint. Click to download.

- Datasets.rar 